langages, information, médiations

$54 \mid 2020$

Les images au cœur des rapports sociaux. Vers de nouveaux régimes de représentation et de visibilité ?

\title{
Victimes de violences conjugales face aux campagnes institutionnelles entre ventriloquie, injonctions et paradoxes
}

Victims of Domestic Violence Confronted with Institutional Campaigns:

Ventriloquism, Injunctions and Paradoxes

\section{Giuseppina Sapio}

\section{(2) OpenEdition}

Journals

Édition électronique

URL : https://journals.openedition.org/edc/10041

DOI : 10.4000/edc. 10041

ISSN : 2101-0366

Éditeur

Université de Lille

Édition imprimée

Date de publication : 1 juillet 2020

Pagination : 53-70

ISBN : 978-2-917562-23-9

ISSN : $1270-6841$

Référence électronique

Giuseppina Sapio, «Victimes de violences conjugales face aux campagnes institutionnelles entre ventriloquie, injonctions et paradoxes », Études de communication [En ligne], 54 | 2020, mis en ligne le 02 janvier 2022, consulté le 07 janvier 2022. URL : http://journals.openedition.org/edc/10041; DOI : https://doi.org/10.4000/edc.10041 
Victimes de violences conjugales

face aux campagnes institutionnelles

entre ventriloquie, injonctions

et paradoxes

Victims of Domestic Violence

Confronted with Institutional

Campaigns: Ventriloquism,

Injunctions and Paradoxes 
Résumé / Abstract

L'article est une restitution problématisée d'un terrain de recherche mené en Italie dans un centre anti-violence accueillant des femmes victimes de violences conjugales. L'étude s'inscrit dans une approche sémio-pragmatique, enrichie par les apports de l'interactionnisme symbolique, et s'appuie sur un dispositif de focus group au sein duquel les femmes ayant subi des violences ont été confrontées aux représentations que les campagnes institutionnelles véhiculent d'elles. L'analyse consiste en une mise en dialogue entre le corpus médiatique et la parole des enquêtées faisant émerger les effets de "ventriloquie ", les injonctions et les paradoxes qui caractérisent le traitement médiatique de ce problème public.

Mots-clés : violences conjugales, violences de genre, communication institutionnelle, campagnes institutionnelles, féminicide, sémio-pragmatique, ethnologie, subjectivation.
This article presents the results of a fieldwork study conducted in Italy in an anti-violence center for female victims of domestic abuse. Based on a semio-pragmatic approach combined with symbolic interactionism (in the form of focus groups), the study explores how victims of domestic violence react to representations of their own situation of victimhood in institutional media campaigns against domestic abuse. The creation of a dialogue between the media corpus and women's testimonies brings to light the effects of institutional "ventriloquism " as well as the injunctions and paradoxes that characterize media coverage of this public problem.

Keywords: domestic violence, gender-based violence, institutional communication, institutional campaigns, femicide, semio-pragmatics, ethnology, subjectivation. 
En 1974, la militante Erin Pizzey rassemble de nombreux témoignages de femmes victimes de violences conjugales dans un ouvrage intitulé Crie moins fort, les voisins vont t'entendre (Pizzey, 1975). Dans la préface, Benoîte Groult rappelle le silence - « un silence de mortes » pour reprendre le titre français de l'ouvrage de Patrizia Romito (2005) - dans lequel sont souvent plongées ces femmes, qui « se sont tues trop longtemps, par pudeur, par orgueil ou par peur » et auxquelles on se doit de "donner les moyens de réagir et de [recouvrer] une dignité élémentaire » (Groult, 1975, 14), pour lesquelles on se doit de crier.

De nombreux changements sont intervenus depuis les années 1970 en Europe et aux États-Unis, et le phénomène des violences faites aux femmes initialement appréhendé à travers le paradigme militant et féministe (Delage, 2017, 113-167) - jouit aujourd'hui d'une reconnaissance institutionnelle et politique. Or, malgré son statut d'évidence, le problème des violences conjugales prend une "forme " (notamment à travers les médias) qu'il faut interroger, celle-ci déterminant "les types de solutions ou de réponses qui devront lui être apportées, les acteurs ou groupes d'acteurs qui devront intervenir dans leur mise en œuvre, ainsi que les valeurs au nom desquelles l'action publique doit être engagée » (Gilbert et Henry, 2012, 43). En d'autres termes, l'existence de campagnes institutionnelles ne nous dédouane pas d'un devoir d'analyse prenant pour objet l'« ordre du discours » (Foucault, 1971, 12). Ainsi, de quelles négociations et de quels compromis (Gilbert et Henry, 2012) les représentations institutionnelles des femmes victimes de violence sont-elles le produit ? En nous situant dans une perspective foucaldienne, nous postulons que ce qui a l'air de constituer « un paysage acceptable est, en fait, le résultat de toute une série de luttes, de conflits, de dominations, de postulats " (Foucault, 1981, 750), et se doit d'être interrogé. Nous nous intéressons donc à la dimension politique du problème des violences conjugales à travers leur médiatisation. Or, si « la politisation d'un problème ne renvoie pas à une prise de position politique visà-vis d'un problème, mais au déconfinement de son traitement par ceux qui en sont considérés comme les spécialistes ou les professionnels » (Gilbert et Henry, 2012, 54), notre position est - tout en incluant cette perspective - plus proche d'un intérêt pour la " politisation des subjectivités » entendue au sens d'Elsa Dorlin $(2017,17)$. Ainsi, notre étude s'intéresse au potentiel subjectivant des discours institutionnels.

La contribution au sujet de Myriam Hernández-Orellana et Stéphanie Kunert, auteures de l'ouvrage Quand l'État parle des violences faites aux femmes (2014) est à cet égard essentielle. Elles y pointent les limites et les contradictions de la communication institutionnelle en France et ce à partir de l'analyse d'un corpus de campagnes gouvernementales. Les femmes y sont prioritairement appréhendées en tant qu'« actants » d'une communication qui leur est directement adressée et leur matérialisation en tant qu'« acteurs » se fait à travers l'exploitation, par les auteures, des résultats de l'enquête ENVEFF (Fougeyrollas-Schwebel et Jaspard, 2008). Inspirée par leur démarche, nous avons souhaité établir un dialogue entre les représentations des femmes victimes de 
violence - « actants » ou « public modèle » pour reprendre la définition d'Umberto Eco (1979) - et la réception par des femmes maltraitées de ces mêmes images. Plusieurs questions ont alors émergé : que se passe-t-il lorsque les images rencontrent leurs destinataires ? Qu'est-ce que les images leur font et qu'est-ce que les femmes font de ces images ? À partir du visionnage des campagnes institutionnelles, les femmes parviennent-elles à inscrire leurs histoires personnelles dans les contours plus larges d'un phénomène systémique et sociétal ? Enfin, la rencontre entre la chercheuse et ses enquêtées produit-elle un savoir opérant et, par conséquent, un pouvoir (une agency)?

\section{1. \\ Une sémiologie pragmatique, critique et politique}

Afin de répondre aux questionnements soulevés, il est indispensable de préciser que notre démarche s'inscrit dans le champ de la sémio-pragmatique (Odin, 2011) et se nourrit des apports de l'interactionnisme symbolique (Goffman, 1975) : pour cela, nous avons mis systématiquement en dialogue l'analyse des campagnes avec la parole des enquêtées, et ce afin d'éviter une séparation nette entre la production (institutionnelle) de représentations de femmes victimes de violence et la réception par ces dernières de ces images. La perspective que nous adoptons est à la fois pragmatique, critique et politique.

Elle est pragmatique dans la mesure où elle refuse une vision éminemment immanentiste des textes qu'elle analyse et se fonde sur la prise en compte des contraintes qui régissent le contexte de production ou de réception d'un message - ce que Roger Odin nomme un " espace de communication ». Ce modèle heuristique " top-down " possède cependant une limite, que l'auteur lui-même reconnaît (Odin, 2011, 141-143), à savoir le degré d'abstraction sur lequel il repose, puisqu'il consiste à émettre des hypothèses quant au fonctionnement d'une situation de communication sans pour autant en interroger les acteurs, étudiés exclusivement dans leur rôle d'actants. Nous nous proposons alors d'hybrider la notion $d^{\prime}$ '« espace de communication " avec un modèle " bottom-up », faisant appel à l'interactionnisme symbolique.

Notre perspective est critique dans le sens qu'elle souhaite déconstruire les représentations médiatiques afin d'identifier le substrat idéologique qui les fonde et qu'elles contribuent à leur tour à fonder, selon un processus circulaire. "La démarche critique n'est bien évidemment pas figée mais elle dépend des affiliations théoriques de chaque chercheurxe ainsi que de la manière dont il / elle [...] conçoit sa propre fonction dans la société " (Sedda, 2017, 3) : ainsi, notre sémiologie s'inspire in primis des travaux barthésiens et, notamment, des Mythologies (Barthes, 1957), dont le contexte politique et historique d'élaboration n'occulte toujours pas, à notre avis, la puissance de leurs intentions. Telle 
que nous la pratiquons, la sémiologie est avant tout une "sismologie », une pratique de la secousse « qui déchire, qui craquelle le nappé, fissure la croûte des langages "(Barthes, 1975, 341). Plus que dans la surface lisse des textes que nous étudions, c'est dans les brèches qu'émanerait le sens : on n'est alors jamais conforté par ce que le texte donne à voir, mais on est constamment animé par l'inquiétude d'entrevoir ce qui ne se voit pas.

Enfin, notre perspective est politique parce qu'elle s'intéresse à la dimension performative des objets médiatiques, appréhendés dans leur capacité à produire des injonctions subjectivantes (Foucault, 1976 ; Butler, 2004 ; Mondzain, 2015). L'accent est mis sur la manière dont les femmes sont «interpellées » (Althusser, 1976) par les campagnes institutionnelles, qui s'adressent à elles tout en les constituant en tant que telles, à savoir exclusivement en tant que victimes des violences masculines. Un tel constat fait partie des résultats de l'étude menée par Hernández-Orellana et Kunert, de laquelle émerge l'énonciation paradoxale de la communication institutionnelle où « le pouvoir d'agir des femmes est quasi inexistant [...] tandis que l'État, énonciateur tutélaire, s'adresse à elles systématiquement à l'impératif (en leur enjoignant notamment de 'briser le silence') » (Hernández-Orellana et Kunert, 2014, 90-91). Au vu de cette analyse, quel éclairage ultérieur les entretiens peuvent-ils apporter ? II ne s'agira pas d'émettre l'hypothèse que l'analyse sémiologique devrait être systématiquement inféodée à une ethnologie, mais d'observer ce que cette dernière peut lui apporter lorsqu'elle intervient.

\subsection{Une ethnologie qui vacille : le G.O.A.P. de Trieste}

Franchir les portes d'un centre antiviolence n'est pas chose aisée. Lorsqu'on y entre en qualité de chercheuse, on est immédiatement saisie par de nombreuses craintes, la plus pressante d'entre elles émanant de l'étrangeté de ce lieu dans lequel on n'a pas sa place et où on s'invite malgré tout, animée des meilleures intentions. La démarche ethnologique vacille, on tâtonne, on tente de se faire discret. On négocie en permanence les réactions, les expressions et la gestuelle, on choisit soigneusement les mots qu'on va prononcer. On est consciente de ne pas partager le même vécu que celles qui fréquentent le centre et pourtant on se rappelle qu'en tant que femme, on a fait d'autres expériences - certes, différentes - de la domination masculine (Bourdieu, 1998), "dans le quotidien, dans l'intimité d'affects de rage enfermés en nous-mêmes, dans la solitude d'expériences vécues de la violence face à laquelle on pratique continûment une autodéfense qui n'en a pas le label » (Dorlin, 2017, 17).

1 Suite à un premier entretien effectué dans un commissariat de Milan autour des violences faites aux femmes, nous avons été mise en contact avec la présidente du centre antiviolence de la ville de Trieste afin de poursuivre notre enquête. 
Notre arrivée à Trieste, en juillet 2018, est l'aboutissement d'un long travail de préparation effectué en amont avec la présidente du centre antiviolence de la ville, nommé G.O.A.P., acronyme de "Groupe d'Opératrices Antiviolence et Projets ". Créé en 1998, le centre est initialement géré par des militantes féministes, soutenues par des associations locales. Un an après, le G.O.A.P. signe la première convention permettant une contribution financière de la part de la mairie qui fournit aussi un appartement faisant office de siège. Le 16 août 2000, la région de Trieste approuve une loi encourageant la réalisation de projets contre les violences envers les femmes ainsi que la mise en place de centres d'accueil, ce qui permet au G.O.A.P. d'ouvrir la première « Maison de refuge ", suivie par une deuxième "Maison d'urgence " ainsi qu'une " Maison de transition ». Les trois dispositifs diffèrent sur le plan du fonctionnement et de la durée de permanence des femmes. En effet, les deux premières se caractérisent par le fait que leur emplacement est secret (puisqu'elles accueillent des femmes en situation de danger) et que la durée du séjour est limitée (six mois pour la "Maison de refuge "; quarante-cinq jours pour celle " d'urgence ") ; alors que la "Maison de transition » héberge des femmes qui ne sont pas ou plus dans une situation de danger imminent et assure la transition entre la sortie du foyer familial et l'entrée dans une vie professionnelle active et indépendante. Contrairement aux précédentes, I'adresse de cet établissement est connue et le séjour peut durer jusqu'à un an. En 2018, le G.O.A.P. enregistre l'arrivée de 305 femmes et compte sur les financements provenant de la région, des institutions locales, d'appels d'offres et d'associations privées. Le centre se compose actuellement d'une équipe de professionnelles ayant des formations variées (psychologues, militantes, sociologues, spécialistes en matière de santé publique). Après une visite des locaux du centre, nous avons mis en place trois focus groups, qui ont duré en moyenne une heure et trente minutes : le premier avec les professionnelles du centre et les deuxièmes avec deux groupes de femmes.

2.

\section{Des actants aux acteurs, et vice-versa}

Les sept femmes ${ }^{2}$ que nous avons rencontrées ont été préalablement sollicitées par la présidente du centre, qui leur a présenté les principes de l'enquête en jouant ainsi un rôle de médiation essentiel. Elles occupent des

2 Nous rendrons compte uniquement de l'un des deux focus groups que nous avons réalisés avec les femmes victimes de violences conjugales. Le choix de ne pas convoquer les résultats relatifs aux deux autres focus groups répond à l'exigence de pouvoir restituer la parole de ces femmes tout en détaillant leurs échanges et interactions. En d'autres termes, nous souhaitions restituer la richesse de l'expérience ethnologique de la manière la plus exhaustive possible, et ce dans les limites inévitablement imposées par le format rédactionnel propre à un article. 
positions sociales très différentes les unes des autres : maîtresse d'école, chômeuse, femme au foyer, entrepreneuse, employée dans le privé. Sur le plan générationnel, le groupe est aussi diversifié - d'une jeune mère de 24 ans à une femme de 68 ans - et six de ces femmes sont mères (dont deux ont un enfant en situation de handicap). La durée de leurs relations avec les hommes maltraitants est très variable (de quelques années jusqu'à 45 ans de mariage) ainsi que la durée de la fréquentation du G.O.A.P. (de quelques mois à plusieurs années).

Le focus group ${ }^{3}$ s'est déroulé dans la salle de réunion du centre et, après une présentation rapide de chaque participante - libre d'évoquer les éléments de son histoire qu'elle jugeait les plus pertinents -, nous avons sollicité leur avis quant au rapport qu'elles entretiennent, en général, avec les médias. Intentionnellement vague, cette question avait l'objectif de favoriser la circulation d'une parole personnelle autour des représentations des violences conjugales, sans s'appuyer sur des exemples précis mais en laissant aux femmes le soin d'évoquer ceux qui leur paraissaient les plus pertinents. Dès le début, les interactions ont été vives et critiques, la prise de parole s'est effectuée de manière fluide, et ce tout au long de la rencontre, avec une distribution assez égale des interventions, à l'exception d'une femme restée en retrait la plupart du temps, bien qu'elle ait suivi attentivement tous les échanges, en approuvant certaines interventions par des hochements de tête.

\subsection{Qu'est-ce que les médias font aux victimes?}

Plusieurs médias ont été évoqués, dont la presse, les séries TV, les films, les docu-fictions et les publicités commerciales avec une approche souvent critique impliquant indirectement la reconnaissance d'une performativité des images et des textes médiatiques. Toutes les enquêtées déploraient l'absence de représentations des violences psychologiques, contrairement aux violences physiques qui sont, selon elles, médiatiquement sur-représentées : «Souvent on réduit le phénomène aux coups, mais il y a beaucoup d'autres choses. La violence, ça dépasse tout ça et la communication est souvent centrée sur la violence physique ». Elles soulignaient ainsi le danger réel constitué par ces représentations qui, en véhiculant un stéréotype de femmes étant exclusivement victimes de coups, risquent d'entraver le processus de dénonciation de celles qui subissent des violences psychologiques tout aussi graves : "Pour le policier, la violence psychologique n'existe pas et, quand il reçoit quelqu'un qui veut porter plainte pour ça, il a l'impression qu'il perd son temps parce que, concrètement, on ne voit rien. Si tu as un cocard ou un bras cassé, alors ils vont considérer l'affaire ". La critique des médias émane d'un sentiment d'injustice que les femmes semblent éprouver face à des récits dans lesquels

3 Les échanges se sont déroulés en italien, ainsi les verbatim reportés dans le texte ont été traduits par l'auteure. 
les hommes sont souvent dépeints comme des sujets mentalement instables (Sapio, 2020), ce qui a pour effet de les affranchir de leurs responsabilités :

«On présente les hommes comme des psychopathes, fous, ou du moins comme des individus mentalement fragiles, dont on comprend qu'ils vont vite vriller. Mais non! Car toutes celles qui sont ici étaient avec des hommes auxquels elles faisaient confiance, ils étaient normaux ! ».

À cet égard, l'une d'entre elles évoque le cas d'un article ${ }^{4}$ paru dans le quotidien L'Avvenire au sujet d'une affaire survenue en mai 2018 et ayant reçu une attention médiatique importante dans le pays. Le texte fait l'objet d'une critique virulente de la part de l'enquêtée : "C'est un article terrifiant, c'était un véritable hymne à cet homme qui a tué sa femme et sa fille... et la journaliste emphatise la figure de cet homme en oubliant complètement les deux femmes [...]. Un homme qui lit cet article, ça lui donne du courage ». Au-delà du contenu, l'un des éléments les plus problématiques réside, selon l'enquêtée, dans le genre de l'instance énonciative du texte : les propos sont d'autant plus graves qu'ils proviennent d'une journaliste qui ne se montre pas solidaire des victimes et qui, au contraire, hérö̈se ${ }^{5}$ le coupable : "Si c'est une femme qui écrit, cela est encore plus douloureux pour moi ». Le journal, dont l'inspiration catholique est notoire en Italie, constitue l'occasion pour les enquêtées de pointer la responsabilité de la religion dans la fabrication d'un idéal de famille dont les injonctions et les valeurs sacrificielles sont contraignantes. Elles regrettent également le fait d'être tenues pour responsables de l'échec du modèle familial lorsqu'elles décident de se séparer de leur conjoint violent : "Quand tu veux le quitter, on te dit souvent 'Tu vas détruire ta famille'. Mais, quelle famille? Si un homme t'humilie, te maltraite, ce n'est plus une famille ». En raison des injonctions culturelles et sociales véhiculées, entre autres, par les médias, les femmes se retrouvent au centre d'un double processus culpabilisant : celui qu'elles subissent et celui qu'elles engendrent elles-mêmes en s'auto-culpabilisant (Romito, 2005, 70-76).

4 Martina Corradi, "L'ultima battaglia di un uomo », L'Avvenire, 22 mai 2018. Lien : https://www.avvenire.it/opinioni/pagine/l-ultima-battaglia-di-un-uomo (consulté le 19 décembre 2019).

5 Sans vouloir produire une analyse de cet article, nous souhaitons souligner certaines formulations qui nous paraissent particulièrement problématiques : à titre d'exemples, le coupable y est désigné, à deux reprises, comme un " pauvre homme ", au sens de " malheureux ", ou comme un "soldat » qui serait « tombé » lors d'une " terrible bataille», en référence aux sept heures d'hésitation passées sur le viaduc d'une autoroute, avant de se jeter dans le vide. 


\section{3. \\ La « ventriloquie» des campagnes institutionnelles}

Après ce débat préliminaire sur leur rapport aux médias, nous avons proposé aux enquêtées de regarder des campagnes institutionnelles. Composé de quatre vidéos, le corpus a été constitué en fonction d'un critère temporel (diffusion entre 2015 et 2017) et énonciatif, les campagnes émanant de différentes instances (le ministère de l'Intérieur, la police et le 1522, le numéro national d'écoute téléphonique pour les femmes victimes de violences). Enfin, un critère formel a également été adopté, permettant de diversifier les représentations du phénomène, allant des plus caricaturales aux plus fines. Dans une perspective sémio-pragmatique, toutes les campagnes fabriquent un « espace de communication " animé par un mode "discursif ", produisant " un texte argumentatif ", des affects " utilisés pour convaincre " et un énonciateur, réel, " interrogeable en termes d'identité, de faire et de vérité »(Odin, 2011, 112), sans oublier que les discours institutionnels se caractérisent « par des phénomènes de formulations conventionnelles, de sloganisation, de figement, de collocation, de cooccurrence » (Krieg-Planque et Oger, 2010, 92). En étudiant l'interaction entre les destinataires et les images qui leur sont expressément adressées, le recours à la notion métaphorique de "ventriloquie ", élaborée par François Cooren, nous a paru alors opérante, dans la mesure où il s'agissait de « reconnaître l'agentivité de l'interlocuteur tout en montrant comment celui-ci fait aussi parler (souvent implicitement) quelque chose, quelque chose qui se met alors à agir par le biais de sa performance »(Cooren, 2010, 40-41).

3.1. Des chiffres et des femmes : la campagne du ministère de l'Intérieur

La première campagne, réalisée par le ministère de l'Intérieur pour la Journée internationale contre la violence faites aux femmes (25 novembre 2015) s'intitule $100 \%$ contre la violence sur les femmes, dure trente-huit secondes et se compose de neuf plans, accompagnés par un court texte indiquant des statistiques : « $31 \%$ des femmes a subi des violences physiques ou sexuelles »; "Plus de $90 \%$ n'a pas dénoncé la violence subie » ; " 70 \% des victimes de viol ont été abusées par leur partenaire ou ex "; "Les enfants de femmes abusées par leur ex partenaire ont assisté aux violences dans $62,4 \%$ des cas $»$. La vidéo se termine avec le slogan " $100 \%$ contre la violence sur les femmes ", où le pourcentage est remplacé par le mot « Tous ». Les plans, accompagnés d'une musique envoûtante et caractérisés par des couleurs douces et un éclairage apaisant, montrent exclusivement des femmes (de tous âges) en train de s'adonner à des activités variées (courses, ménage, loisirs avec les enfants, trajets dans les transports). L'« homme violent ", matérialisé par la voix off qui, par le ton et le contenu de ses phrases, établit une connexion oxymorique avec les images, s'adresse à un énonciateur individuel «Tu », vers lequel il dirige diverses injonctions : «Tu vas où ?! Où penses-tu aller ?! », " N'ose pas dire un mot » (ton 
virulent) et « Tu es à moi, rien qu'à moi, mon amour » (ton conciliant). La dernière phrase s'adresse à un énonciateur pluriel «Vous », que l'on présume incarner les enfants du couple : "Qu'est-ce que vous avez à regarder, vous ? Au lit !".

Les réactions à cette campagne ont évolué tout au long de l'échange entre les femmes, qui s'est déroulé sans que nous devions intervenir. Le point de départ a été la reconnaissance de la part de l'une des enquêtées du réalisme de la campagne ("C'est très réaliste, c'est ce qui généralement arrive, avec toutes les phases de la violence »), qualité que les autres se sont aussitôt empressées de mitiger :

«Oui, par contre le message est présenté de manière très délicate... ».

"Oui, avec la petite musique..."

"Moi, ça m’a pas secouée, avec sa petite musique, les draps blancs, je ne m'identifie pas avec ce que j'ai vécu... avec les scènes au ralenti ».

Cet échange a ouvert la voie à d'autres critiques, dont celle concernant I'inutilité des chiffres : "À qui s'adresse-t-elle ? À nous ? Ok, les statistiques mais, ça, je connais, je l'ai vécu. On dirait les pubs de Mulino Bianco ${ }^{6}$. Ils auraient pu ajouter 'Nos gâteaux ne contiennent pas d'huile de palme et $30 \%$ de cas de violences conjugales ne sont pas dénoncés' ". Contrairement aux représentations institutionnelles, montrant généralement des femmes taciturnes et abattues, les interventions de nos enquêtées ont souvent été teintées d'ironie et d'auto-ironie, des affects résultants certainement du processus de réhabilitation entamé avec le centre. Ici, le lien ironique établi entre la campagne et une publicité commerciale semble dissimuler la blessure que la mobilisation des chiffres produit chez cette femme, qui voit son expérience de la violence en quelque sorte déshumanisée.

Sollicitées quant à l'absence d'un homme à l'écran, les enquêtées avouent d'abord ne pas avoir remarqué cet aspect, mais reconnaissent tout de même le réalisme des propos prononcés par la voix off masculine. Pour reprendre la métaphore de la " ventriloquie » de François Cooren (2010), la voix incarne, d'une part, l'énonciateur individuel que chacune a connu et rencontré : " Je ne sais pas si ça vous est arrivé aussi, mais le mien me disait toujours qu'il m'aimait, il se ramenait avec des fleurs... ". D'autre part, elle se fait porte-parole d'une culture qui dépasse les contours de l'espace domestique et s'inscrit dans ceux plus larges de la société italienne : "De manière générale, on a une culture patriarcale et machiste, dans laquelle tu es passive, tu attends ton prince, qui te prend et te jette et toi, tu es toujours là en train de l'attendre ».

6 Célèbre marque de gâteaux - qui oriente sa communication vers l'image idéalisée d'une famille réunie et sans conflits - souvent mobilisée, dans les conversations informelles, pour critiquer une vision ou une représentation trompeuse, voire hypocrite, des relations interpersonnelles. 


\subsection{Victimes d'un stéréotype : la campagne de la police}

La deuxième campagne a été réalisée le 14 février 2017 par la police, dure une minute et dix secondes, se compose de neuf plans et est entièrement tournée en noir et blanc, à l'exception du dernier plan qui est en couleurs. Elle ne montre pas les hommes maltraitants mais se focalise exclusivement sur les victimes - trois à l'occurrence, plutôt jeunes et ayant des caractéristiques communes : habits noirs, maquillage défait, regard sombre, posture recroquevillée, attitude inquiète. Sur le plan sonore, trois éléments concourent à la construction d'une ambiance angoissante : d'abord, les notes aiguës d'un piano jouant une musique inquiétante ; ensuite, le cliquetis sec (proche du bruit d'une gifle) d'un interrupteur en correspondance de certains cuts; enfin, une voix off féminine, s'exprimant à la première personne et incarnant progressivement le ressenti des femmes montrées à l'écran : " II m’a giflée. Peut-être, c'est de ma faute "; "Je croyais qu'il pouvait changer, je me trompais "; " Je crains qu'il ne soit désormais trop tard pour fuir cet amour malade ". Si les deux premières phrases sont prononcées avec un ton plaintif, la dernière est proférée avec un ton dramatique. Les corps de ces femmes vêtues de noir sont littéralement engloutis, à l'exception de leurs visages, par le fond noir des plans larges, alors que l'éclairage latéral crée des ombres denses contribuant davantage à l'esthétique « expressionniste » de la campagne.

Sur l'avant-dernier plan composé d'un fond sombre, une autre voix off féminine intervient, cette fois-ci plus confiante et déterminée, qui prononce un ensemble de propositions subordonnées hypothétiques (" $S^{\prime}$ il t'humilie "; "S'il te menace, toi et tes enfants "; "S'il t'isole et t'insulte "; "S'il te frappe »; "Si le cœur bat plus vite sous le coup de la peur ») complétées par la proposition principale «Ce n'est pas ça l'amour ». Dans le dernier plan en couleurs, l'institution policière s'incarne en une femme, débout et en uniforme, s'adressant à un énonciateur collectif «Vous » ("Vous n'êtes pas seules »).

La première réaction des femmes à cette campagne a été un éclat de rire collectif. Les enquêtées déploraient unanimement l'image que celle-ci véhicule des victimes et insistaient sur le fait que, tout au long des violences subies, elles se sont toujours efforcées de feindre un bonheur inexistant. Les énergies investies non seulement pour résister aux abus, mais aussi pour " garder la face " (Goffman, 1973, 64) constituent, selon elles, la clé de voûte pour comprendre leur état de dissociation personnelle :

"On montre toujours des femmes faibles, mais nous, nous sommes des personnes qui, à l'apparence, font apparaître tout plus léger, qui rigolent ou rient le plus "; "Je justifiais constamment ses comportements aux yeux de tout le monde, j'étais son avocate "; et, encore, "II faudrait montrer une femme à l'apparence joyeuse, mais avec un background et une voix off qui dévoile une réalité plus sombre, par rapport à laquelle elle se justifierait en disant qu'elle doit pourvoir aux besoins de ses enfants ». 
Interrogée quant à l'existence d'un stéréotype de la victime, cette dernière répond par l'affirmative en mimant une attitude de repli ; tandis qu'une autre souligne le risque qu'une telle vision peut engendrer : "Tu peux te permettre de faire la victime seulement si tu es comme ça, habillée en noir, avec des grosses cernes ". La légitimité de la parole de la victime dans l'espace public est, selon elle, aussi déterminée par ce type de communication erronée. Pour reprendre la métaphore de François Cooren (2010) sur la ventriloquie, on pourrait avancer que, en pensant "faire parler » les victimes à travers ses représentations, l'institution devient, à son tour, le pantin d'une culture dans laquelle circule une norme tacite définissant une image stéréotypée des victimes de violences conjugales.

Néanmoins, cette femme reconnaît tout de même que le slogan est bien choisi, en recevant l'approbation de toutes les autres, puisqu'il est axé sur la peur que l'on éprouve au quotidien : "Cette phrase est très juste parce que c'est ce qui arrive ou, du moins, c'est ce qui m'est arrivé avec les violences psychologiques. Après le lavage du cerveau, tu es lobotomisée, du coup on t'induit à avoir des comportements qui ne sont pas basés sur ce que tu veux, mais sur ce que lui [elle insiste] il veut, donc si tu ne fais pas exactement ce qu'il veut, tu es dans la merde. C'est ça la peur. Mais ce n'est pas facile à comprendre par ceux qui ne l'ont pas vécu ». Les femmes concordent sur un sentiment partagé, celui de l'« assiégement », qui nous renvoie à l'efficacité de la voix off masculine de la campagne précédente traduisant, plus que la voix féminine de cette campagne, leur état d'esprit. À cet égard, l'une d'entre elles avoue qu'elle avait désormais intériorisé la voix de son compagnon : « Je me suis rendu compte que, même quand il n'était pas là pendant des journées entières, à cause du travail... [une femme l'interrompt pour lui suggérer 'Tu allais mieux !']. Non ! J'avais une voix intérieure, la sienne, j'avais carrément des dialogues imaginaires avec lui ». La voix off masculine demeure ainsi, selon elles, un bon moyen pour " mettre en scène » un état de contrôle permanent.

\subsection{Le paradoxe de la victime : la campagne du 1522}

Les deux dernières campagnes ont été réalisées en novembre 2017 par le service téléphonique d'écoute 1522 promu par la présidence du Conseil. Diffusées en proximité de la date du 25 novembre, ces deux vidéos durent environ une quarantaine de secondes, se composent respectivement de dixneuf et dix-sept plans et fonctionnent selon les mêmes modalités, à savoir un montage alterné opposant les plans dans lesquels la victime discute avec une amie à ceux où on la voit en présence de l'homme maltraitant. Dans la première, la protagoniste raconte avoir passé une belle soirée avec son compagnon à l'occasion de leur anniversaire de mariage ; cependant, son récit est aussitôt contredit par les images, dans lesquelles elle se fait agresser. Dans la deuxième, une jeune femme explique à son amie la raison qui l'a empêchée de la rejoindre la veille. Ici aussi, les images dévoilent le mensonge de la victime prétextant que sa voiture ne démarrait pas alors qu'on découvre la présence oppressante de 
son ex-partenaire qui l'accable de textos et l'agresse lorsqu'elle descend dans le parking. La conclusion est la même : les deux amies-témoins saisissent le mensonge et tendent aux victimes un téléphone mobile pour qu'elles puissent composer le numéro d'écoute. Les vidéos se terminent avec un slogan (" La violence a beaucoup de mots, nous on veut entendre les tiens. Débloque le courage, contacte le 1522 ») et se caractérisent par une esthétique épurée, appuyée par une musique jouée au piano, par une très faible profondeur de champ et par un éclairage doux, éléments qui soulignent davantage la violence des hommes maltraitants.

Après le visionnage, le retour des femmes est globalement positif, et ce pour une raison précise, à savoir la mise en scène du clivage que les victimes vivent tout au long de leur relation, tiraillées entre les abus subis et la nécessité de « garder la face » vis-à-vis des autres :

«On voit qu'elle doit inventer des excuses, ça m'est arrivé tellement de fois d'avoir un rendez-vous avec des amis et de ne pas pouvoir y aller parce qu'il s'énervait... et puis je racontais toujours des bobards aux autres ».

"La deuxième m'a émue parce qu'elle montrait ce que j'ai vécu et que je n'ai probablement pas encore réélaboré ".

" Ça montre le fait qu'on vit dans l'apparence, que tout va bien, jusqu'au moment où tu finis par comprendre. Mais avant tu t'acharnes parce que tu veux ce que tu n'auras plus jamais, ce que tu avais avant, au début de I'histoire, et qui parfois n'a duré qu'un mois ».

Le potentiel performatif de ces vidéos est reconnu et valorisé au point qu'une enquêtée suggère qu'« une femme qui voit ça se dit 'Mince, c'est mon dîner d'hier soir' ». Les femmes soulignent également le fait que la parole "paradoxale » qui caractérise le cycle de la violence devrait être montrée davantage, à travers une alternance des aspects positifs et négatifs de la relation. Bien qu'inconfortable, une représentation "paradoxale » des victimes, devenant malgré elles - les complices de leurs partenaires, semble nécessaire selon les enquêtées : "Je trouve incisif le fait de montrer que, de l'extérieur, tout paraît tranquille et que, donc, même avec une amie, tu voudrais lui raconter des choses, mais tu ne le fais pas parce que tu as honte ".

\section{4. \\ Mon amour, si tu me tues, qui frapperas-tu après ? : une campagne paradoxale}

La campagne du 1522 a été l'occasion pour les enquêtées d'évoquer les raisons du lien de " connivence " établi avec leurs bourreaux : les violences reçues, la relation d'intimité, les injonctions morales et sociales qui les 
orientent vers la préservation du couple ou de la famille. Une femme affirme que le sentiment négatif de dépendance vécu vis-à-vis du partenaire finissait par prendre la forme, "positive » et tout aussi paradoxale, du désir de rester avec lui : "Dans certains cas, on s'enfonce dans cette spirale de violence qui devient une toile d'araignée, dans laquelle tu n'es qu'un minuscule insecte à la merci de cet homme. Au fond de toi, tu espères que cette situation ne change pas, toi aussi tu veux que ça ne change pas parce que - paradoxalement [elle souligne] - tu as besoin de cette personne ". Un tel paradoxe est aussi évident lorsque les femmes déplorent l'omerta de certains voisins : "Personne n'a appelé la police quand il me frappait, ils faisaient comme si de rien n'était. Ceci dit, moi aussi je faisais quelque chose - qui ne servait à rien, hein : quand il commençait à bouillir, je fermais tous les volets et les stores, toutes les fenêtres, toutes les portes ".

Selon les enquêtées, la dimension " paradoxale » de la relation violente devrait être centrale dans les campagnes institutionnelles, qui seraient ainsi plus réalistes et efficaces. À cet égard, une femme propose aux autres de considérer une campagne ${ }^{7}$ que nous n'avions pas prévu de projeter, car nous pensions qu'elle aurait pu déstabiliser certaines d'entre elles. Il s'agit d'une vidéo de cinq minutes environ dont le slogan, "Mon amour, si tu me tues, qui frapperas-tu après ? ", avait été âprement critiqué par les médias et les instances politiques. La protagoniste, interprétée par Rosalia Porcaro, une actrice comique connue du grand public, excuse systématiquement les agissements de son mari en alléguant des raisons absurdes. Elle dit par exemple avoir été " maquillée " par son homme, en référence aux marques sur son visage, et elle affirme que si, malencontreusement, l'équipe de foot de son mari perd un match, c'est nécessairement elle qui fera l'objet de sa colère, le téléviseur leur ayant coûté trop cher pour qu'il l'abîme. Toute la vidéo est ponctuée par la répétition du slogan et l'épilogue laisse entendre qu'elle a finalement été tuée. Nos réticences étaient donc liées non seulement au contenu des répliques mais également au ton ironique, voire presque comique, avec lequel elles sont prononcées.

Cependant, et contrairement à nos attentes, le message est très bien accueilli par nos enquêtées, dont une finit par dire que c'est «l'apothéose du message ». La femme ayant proposé cette campagne confirme que, dans son entourage, le message avait eu une réception très négative, alors qu'elle le trouvait incisif en raison de sa capacité à souligner la dimension paradoxale de la relation de dépendance avec les hommes maltraitants : "Tout le monde le trouvait horrible, ils étaient révoltés, mais moi je l'aime bien. Quand je l'ai vu la première fois, je me suis effondrée, mais le message était selon moi efficace à propos des violences faites aux femmes. Tous les autres me disaient que

7 Une campagne écrite et dirigée par Corrado Ardone, diffusée en novembre 2017, produite par Maxima Film. Plusieurs instances régionales et locales, comme la région Campanie et la mairie de Naples, ont contribué à la réalisation. Lien : https://youtu.be/67ZieiSS9rE (consulté le 19 décembre 2019). 
c'était inacceptable, que c'était contre les femmes, que le message desservait la cause des femmes ". Cet exemple nous éclaire non seulement quant aux différences de réception que les mêmes images peuvent engendrer mais également sur le fait que leur coefficient performatif, à savoir leur capacité à agir sur les publics, peut varier considérablement. La performativité des images est fonction du degré de d'implication des sujets qu'elles interpellent. Cet exemple de "ventriloquie " où, en s'indignant, on présume donner la voix aux victimes alors que celles-ci - à l'échelle de notre échantillon - n'ont pas le même ressenti, nous montre combien la consultation de ces dernières pour la réalisation d'objets médiatiques "sensibles ", comme les campagnes institutionnelles, devient nécessaire.

\section{Conclusion : penser le collectif, se penser en collectif}

Si la situation d'entretien nous a permis d'observer comment les femmes répondent aux injonctions subjectivantes propres à la communication institutionnelle, elle nous a également montré qu'elle engendre un processus collectif de subjectivation, appuyé par le cadre réhabilitant offert par le centre antiviolence permettant - entre autres - de regarder sous un angle plus critique des représentations qui se veulent consensuelles. L'échange entre les enquêtées s'est caractérisé par l'émergence progressive d'une énonciation particulière : d'une part, le « nous » des victimes qui, en partageant leurs expériences ont pu observer de nombreuses similarités avec le récit des autres ; d'autre part, le " eux » des hommes maltraitants et de celles et ceux qui les ont culpabilisées lorsqu'elles ont voulu les quitter.

Les campagnes institutionnelles ainsi que la situation d'entretien contribuent à donner aux femmes le sentiment que leurs histoires s'inscrivent dans un phénomène systémique et structurel dont l'ampleur dépasse les contours de la souffrance individuelle : "Rien que le fait que nous soyons ici, réunies, en train de partager nos vécus, ça veut déjà dire qu'un changement culturel est en cours ». Par ailleurs, il est utile de rappeler qu'aucune des enquêtées n'a explicitement dit avoir été marquée par l'une des campagnes lorsqu'elle était avec son compagnon violent. Nos résultats sont donc strictement corrélés aux conditions de réception particulières dans lesquelles les campagnes ont été visionnées, à savoir rétrospectivement, une fois que le travail de réhabilitation a été entamé avec le centre. Ceci étant, les deux dispositifs (la situation d'entretien et le cadre offert par le centre) permettent, potentiellement, de penser le collectif et de se penser en collectif, en esquivant ainsi le risque d'une « trop forte personnalisation et [...] personnification de la souffrance » et en contribuant à " 'socialiser' la souffrance, [à] la dépsychologiser, notamment en évoquant la similitude des contenus de vie, des épreuves personnelles, et des causes envisagées par les malheureux »(Huet, 2016, 131-132). Une fois que les vio- 
lences conjugales ne sont plus perçues comme le fait d'une relation spécifique, mais s'inscrivent dans un phénomène plus vaste, la subjectivation des femmes passe également par la reconnaissance du caractère aberrant de la demande que la société formule à leurs égards en postulant que, en l'absence d'une transformation culturelle et sociale du système de la domination masculine, elles se doivent d'opérer ce changement. II semble alors que les différentes injonctions adressées aux femmes dans les campagnes institutionnelles s'appuient sur une naturalisation de la violence masculine : "On part souvent du présupposé que la violence chez les hommes est innée et que tu ne peux pas la changer. Alors, si tu ne peux pas changer l'homme, tu te dois d'informer la femme pour qu'elle parvienne à distinguer la violence et à ne pas entrer dans cette spirale », ce qui revient à affirmer que " la femme est responsable de tout ». L'une d'entre elles, la plus silencieuse tout au long de l'échange, intervient alors pour dire d'un ton ferme qu'« il faudrait une campagne pour eux, où on leur dit 'Réveillez-vous !' ».

Nous terminerons en précisant que si, d'une part, nous avons pu apporter aux enquêtées un éclairage sur les spécificités de la communication institutionnelle, en leur signalant certains éléments qui nous paraissaient problématiques, celles-ci nous ont offert, selon un processus circulaire, un autre savoir, "situé » pour reprendre les mots de Donna Haraway $(2007,118)$, qui - comme tout savoir - est inévitablement un pouvoir : celui de changer de perspective, de concevoir une autre manière de voir. En empruntant leurs yeux, l'impensé et l'indicible du slogan "Mon amour, si tu me tues, qui frapperas-tu après ? » devient alors finalement acceptable. 
Bibliographie

Althusser L. (1976). «Idéologie et appareils idéologiques d'État. Notes pour une recherche $»$. In Positions (1964-1975), Paris, Les Éditions sociales, p. 67-125.

Barthes R. (1957). Mythologies, Paris, Gallimard.

Barthes R. (1995 [1975]). « Brecht et le discours : contribution à l'étude de la discursivité ". In L'Autre Scène, vol. 8-9 repris dans Fuvres complètes III, Paris, Seuil, p. 340-351.

Bourdieu P. (1998). La domination masculine, Paris, Seuil.

Butler J. (2004). Le pouvoir des mots : discours de haine et politique du performatif, Paris, Éditions Amsterdam.

Cooren F. (2010). « Ventriloquie, performativité et communication ou comment fait-on parler les choses ". In Réseaux, vol. 163, n 5, p. 33-54.

Daligand L. (2016). Les violences conjugales, Paris, Presses Universitaires de France, coll. "Que sais-je?".

Delage P. (2017). Violences conjugales. Du combat féministe à la cause publique, Paris, Presses de la Fondation nationale des sciences politiques.
Dorlin E. (2017). Se défendre: une philosophie de la violence, Paris, Zones.

Eco U. (1979). Lector in fabula : la cooperazione interpretativa nei testi narrativi, Milan, Bompiani.

Foucault M. (1971). L'ordre du discours, Paris, Gallimard.

Foucault M. (1976). Surveiller et punir : naissance de la prison, Paris, Gallimard.

Foucault M. (1984 [1981]). « L'intellectuel et les pouvoirs. Entretien avec C. Panier et P. Watté ». In Foucault M., Dits et Écrits, t. IV, texte $n^{\circ} 359$, Paris, Gallimard, p. 747-752.

Fougeyrollas-Schwebel D., Jaspard M. (2008). « Représentations de la violence envers les femmes dans le couple : mesures du phénomène Le cas français ". In Santé, Société et Solidarité, $n^{\circ} 1$, p. 109-116.

Gilbert C., Henry E. (2012). « La définition des problèmes publics : entre publicité et discrétion ". In Revue française de sociologie, vol. 53, n² 1, p. 35-59.

Goffman E. (1973). La mise en scène de la vie quotidienne. 1. La présentation de soi, Paris, Éditions de Minuit.

Goffman E. (1975), Les rites d'interaction, Paris, Éditions de Minuit.

Groult B. (1975). "Préface ». In Pizzey E., Crie moins fort, les voisins vont t'entendre, $\mathrm{Pa}$ ris, Des Femmes, p. 7-14. 
Haraway D. (2007). Manifeste cyborg et autres essais. Sciences - Fictions Féminismes, Paris, Exils éditeurs.

Hernández-Orellana M., Kunert S. (2014). Quand l'État parle des violences faites aux femmes, Fontenay-le-Comte, Éditions Lussaud.

Huet R. (2016). « Et si je suis désespéré, à qui dois-je en vouloir?". In Revue française d'éthique appliquée, vol. 2, n² 2, 118-132.

Krieg-Planque A., Oger C. (2010). «Discours institutionnels. Perspectives pour les sciences de la communication ». In Mots. Les langages du politique, vol. 94, p. 91-96.

Mondzain M.-J. (2015). L'image peut-elle tuer?, Montrouge, Bayard.

Odin R. (2011). Les espaces de communication : introduction à la sémio-pragmatique, Grenoble, Presses Universitaires de Grenoble.

Oger C., Ollivier-Yaniv C. (2006). "Conjurer le désordre discursif. Les procédés de 'lissage' dans la fabrication du discours institutionnel ». In Mots. Les langages du politique, vol. 81, p. 63-77.

Pizzey E. (1975). Crie moins fort, les voisins vont t'entendre, Paris, Des Femmes.

Romito P. (2005). Un silenzio assordante : la violenza occultata su donne e minori, Milan, Franco Angeli.
Sapio G. (2020). « L'amour qui hait. La formule 'crime passionnel' dans la presse française contemporaine ". In Semen, vol. 47, p. 23-42.

Sedda P. (2017). « Approche critique : quelle appropriation par les SIC ? ». In Revue française des Sciences de l'Information et de la Communication, vol. 11, p. 1-13. 